\title{
Role of Laboratory in Diagnosing Fungal Infections
}

\author{
Liz Mary Paul ${ }^{1}$, Ajay V ${ }^{2}$
}

\begin{abstract}
The laboratory plays a crucial role in diagnosing fungal infections that are often underdiagnosed. Identifying invasive fungal infections in the immunocompromised population requires a high index of suspicion and choice of diagnostic methods is of utmost importance. This article aims to describe the various methods in a nutshell and hence explain how an integrated approach helps in accurate diagnosis which will benefit the patient. It also illustrates a few interesting case reports along with their laboratory findings.
\end{abstract}

Keywords: Fungal infections, Laboratory diagnosis, Potassium hydroxide mount.

Pediatric Infectious Disease (2021): 10.5005/jp-journals-10081-1294

\section{INTRODUCTION}

Fungal infections are an increasing threat to children, although they are considered less common than bacterial infections. However, the truth is mycology testing methods are not easily available in laboratories of developing countries like India probably due to economic constraints or lack of expertise. Hence, diagnosed fungal infections form the tip of the iceberg. Considering global epidemiology, the number of fungal infections is on the rise, major factors responsible for this being host alteration like the growing number of immunocompromised people and the overuse of broadspectrum antibacterial drugs.

Fungi encountered in the clinical laboratory belong to two groups - the yeasts and the filamentous fungi/molds. Examples of yeasts include Candida and Cryptococcus, whereas molds include Aspergillus and Rhizopus. Some fungi exhibit dimorphism, i.e., they exist in both forms under different conditions, e.g., thermal dimorphism of systemic fungi like Histoplasma. Clinical classification of fungi is based on the site of involvement-superficial/ cutaneous mycoses, subcutaneous mycoses, systemic mycoses, and opportunistic mycoses. Superficial/cutaneous mycoses are confined to skin/hair/nails and do not invade deeper tissues. Dermatophytes and fungi causing tinea versicolor, tinea nigra, and piedra are included in this group. Subcutaneous mycoses are confined to subcutaneous tissues; examples being Chromoblastomycosis and Mycetoma. Agents causing systemic fungal infections include Histoplasma, Coccidioides, Paracoccidioides, and Blastomyces. Opportunistic mycoses are seen in patients with a compromised immune system like diabetes, HIV, immunodeficiency syndromes, and malignancies. Invasive fungal infections in children are opportunistic mycoses causing significant morbidity and mortality, especially in hematological malignancies like leukemia, or those who have undergone a hematopoietic stem cell or solid organ transplant. These infections are also reported in premature neonates or those with congenital or acquired immunodeficiency. Examples include Candida, Aspergillus, and Cryptococcus. ${ }^{1-3}$ This review aims to discuss laboratory aspects of these mycoses along with interesting cases published in the literature.

\section{Fungi in the Microbiology Lab- Practical Aspects}

\section{Specimen Collection and Transport}

Appropriate specimen collection and transport is crucial to the recovery of fungi. Slow growing fungi may be easily overgrown
${ }^{1}$ Department of Microbiology, IQRAA International Hospital and Research Centre, Calicut, Kerala, India

${ }^{2}$ Department of Pediatrics, Baby Memorial Hospital, Calicut, Kerala, India

Corresponding Author: Liz Mary Paul, Department of Microbiology, IQRAA International Hospital and Research Centre, Calicut, Kerala, India, Phone: +91 8281490911, e-mail: lizmarypaul87@gmail.com

How to cite this article: Paul LM, V Ajay. Role of Laboratory in Diagnosing Fungal Infections. Pediatr Inf Dis 2021;3(1):33-37.

Source of support: Nil

Conflict of interest: None

by bacteria if transport and processing is delayed. Specimens except skin, hair, nails, cerebrospinal fluid (CSF), and blood may be refrigerated for a short time.

\section{Skin and Subcutaneous Tissue}

Skin scrapings are collected from the peripheral region of suspected Tinea infections using a sterile scalpel blade. The area should be cleaned with saline/alcohol before scraping. Alcohol should be avoided if fungal culture is to be done. Scrapings may be collected on black paper or directly onto the slide. Punch biopsy specimens may be sent for suspected subcutaneous fungal infections.

\section{Hair}

Plucked hair is sent for mycological investigation in suspected Tinea/Piedra. Direct macroscopic examination of hair also provides a clue to the diagnosis.

\section{Nails}

Nails may be discolored/brittle in dermatophytosis, nail clippings from infected areas are sent to the laboratory for investigations.

\section{CSF and Body Fluids}

Body fluids should be sent in a sterile culture bottle. Blood may be sent for automated culture in case of suspected invasive candidiasis. Biopsy/Tissue Specimens

Biopsy specimens from various sites may be sent for fungal staining/ culture; however, special care is to be taken to avoid sending in formalin. Specimens may be sent as such in sterile culture bottle or saline if a delay in transport is anticipated (Figs 1 to 9). 


\section{Direct Microscopic Examination}

\section{Potassium Hydroxide Mount}

Potassium hydroxide $(\mathrm{KOH})$ mount is a very useful direct microscopic examination procedure in diagnostic medical mycology to detect fungal spores or hyphae or yeasts. $10 \% \mathrm{KOH}$ is used for hair and skin, whereas $20 \% \mathrm{KOH}$ is used for nails/hyperkeratotic specimens. An initial examination is done with low power magnification (10X) followed by a higher magnification (40x). Branching hyphae can be seen, characteristics of branching and presence or absence of septations give a clue to the diagnosis. Yeasts may be seen in Candidiasis/Pityriasis versicolor and budding can be noted. Artifacts that may mimic fungal hyphae are lines of the juncture of normal epidermal cells dissolve into branching network, cotton fibers, and synthetic fibers. Modifications of $\mathrm{KOH}$ by adding Parkers ink/eosin have also been applied in laboratories. Refractile, long, smooth, undulating, branching, and septate hyphal filaments with or without arthroconidiospores are seen in dermatophyte involvement. In Candidiasis, hyaline, oval, budding (blastoconidiating) yeast cell forms with or without pseudo-hyphae are observed. Spherical yeast in clusters along with short filaments resembling "banana and grapes" or "spaghetti and meatballs" are typical of Malassezia furfur causing pityriasis versicolor. $\mathrm{KOH}$ mount is a rapid, simple to perform the inexpensive test but it is not highly specific and requires expertise for interpretation. ${ }^{4}$

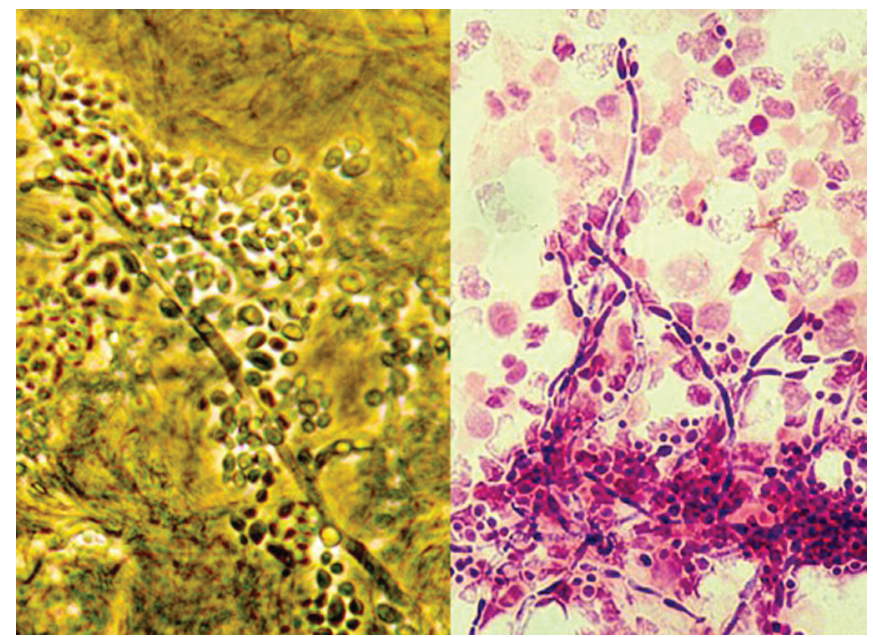

Fig. 1: 10\% KOH mount showing budding yeast cells with pseudohyphae in skin scraping; PAS stained smear showing the same

\section{Gram's Stain}

Gram's stain is a differential stain useful for diagnosing fungal infections like Candidiasis, Cryptococcosis, and systemic fungal infections like Histoplasmosis. Yeast fungi are gram-positive whereas fungal hyphae are usually gram-positive but may show variability. Candida species are seen as gram-positive oval budding yeast cells with pseudo-hyphae. Cryptococcus neoformans is a gram-positive spherical budding yeast. Histoplasma capsulatum var. capsulatum yeast cells are gram-positive, ovoid in shape, and measure $2-4 \mu \mathrm{m}$ in size.

\section{India Ink Stain}

India ink stain is a negative staining technique useful for diagnosing Cryptococcal meningitis. Cryptococci because of its large polysaccharide capsule, exhibit a halo around the cell against the black background created by the India Ink. Budding can also be observed which differentiates it from host inflammatory cells. However, it is insensitive for low fungal burdens, which can be common in persons presenting early after symptom onset or those presenting on antiretroviral therapy. The sensitivity is as low as $42 \%$ with fungal burdens of $<1,000$ colony forming units (CFU)/mL on quantitative CSF culture. ${ }^{5}$

\section{Fungal Special Staining Techniques}

These include Masson Fontana's, Gomori methenamine silver, Wright's and periodic acid Schiff staining techniques. They are used for cytopathology of tissue aspirates and fluids and in histopathology specimens.

Histopathological examination of tissues detects fungal invasion of tissues and vessels as well as the host reaction to the fungus and thus remains an important tool to define the diagnostic significance of positive culture isolates. Fontana-Masson stains fungal cell walls black against a pale pink background, whereas Grocott's modification of Gomori methenamine silver method stains cell walls brown to black against a background of pale green. Hematoxylin and eosin stain is useful for Aspergillus and Zygomycetes as well as yeasts where basophilic yeast cytoplasm is separated from the surrounding tissue by a clear zone corresponding to the cell wall. Aspergillus shows hyaline septate hyphae with acute angle branching, whereas Mucorales show pauci-septate broad ribbonlike hyphae with right-angle branching. Various agents of systemic fungal infections, Pneumocystis, Sporotrichosis, and many other mycoses may be diagnosed with these staining methods along with clinical and culture correlation. ${ }^{6,7}$

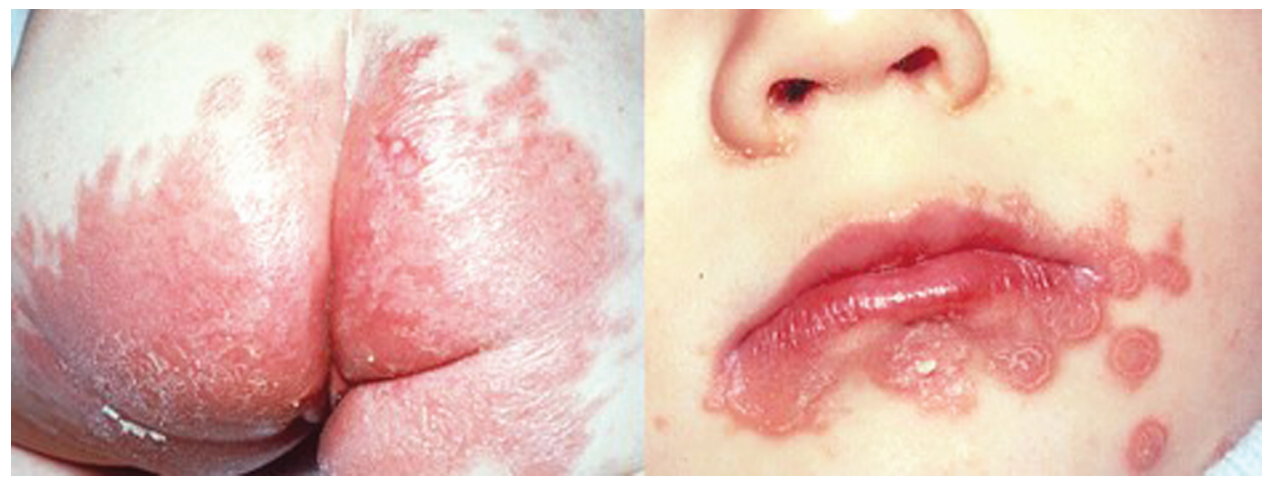

Fig. 2: Nappy rash and perioral candidiasis 


\section{Calcofluor White (CFW) Stain}

It is a fluorescent stain for the rapid detection of yeasts and other fungi. The nonspecific fluorochrome stain is a specific dye for chitin and cellulose and displays fluorescence when exposed to long wavelength ultraviolet and short-wavelength visible light. Interpretation requires expertise, cost, and non-availability of fluorescent microscope limit its use in many laboratories. ${ }^{8}$

\section{Fungal Culture}

A fungal culture is a gold standard method to diagnose fungal infections. However, in some scenarios, saprophytic fungi may grow

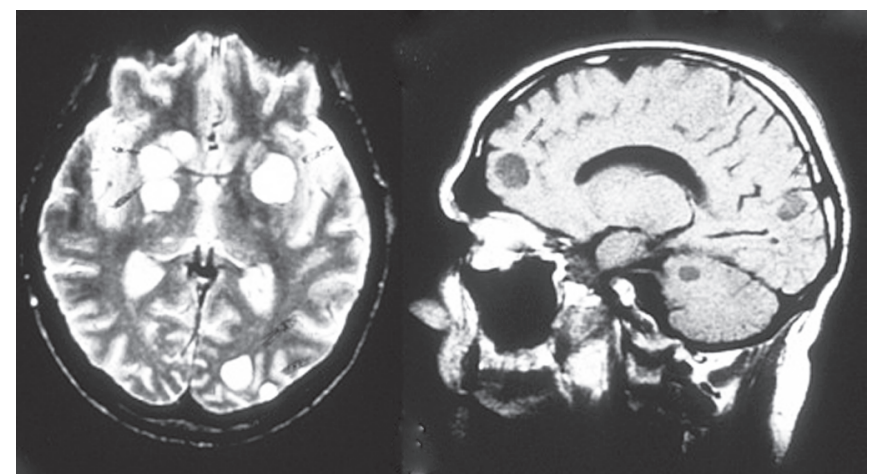

Fig. 3: MRI brain showing cryptococcomas

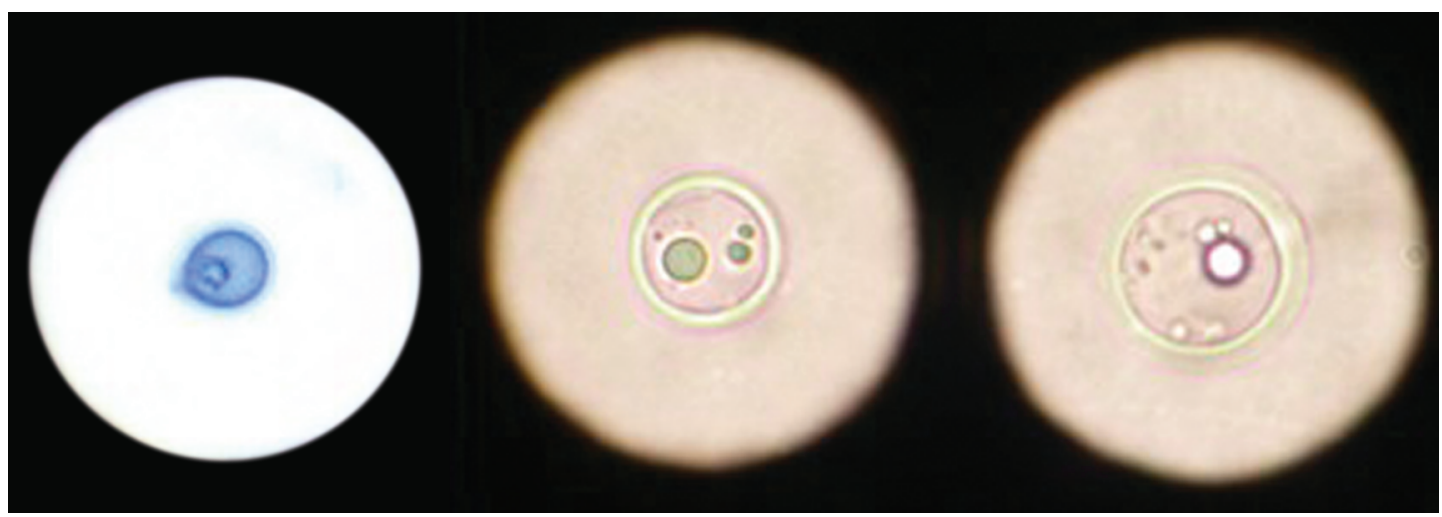

Fig. 4: India ink preparation showing yeast cells with capsule

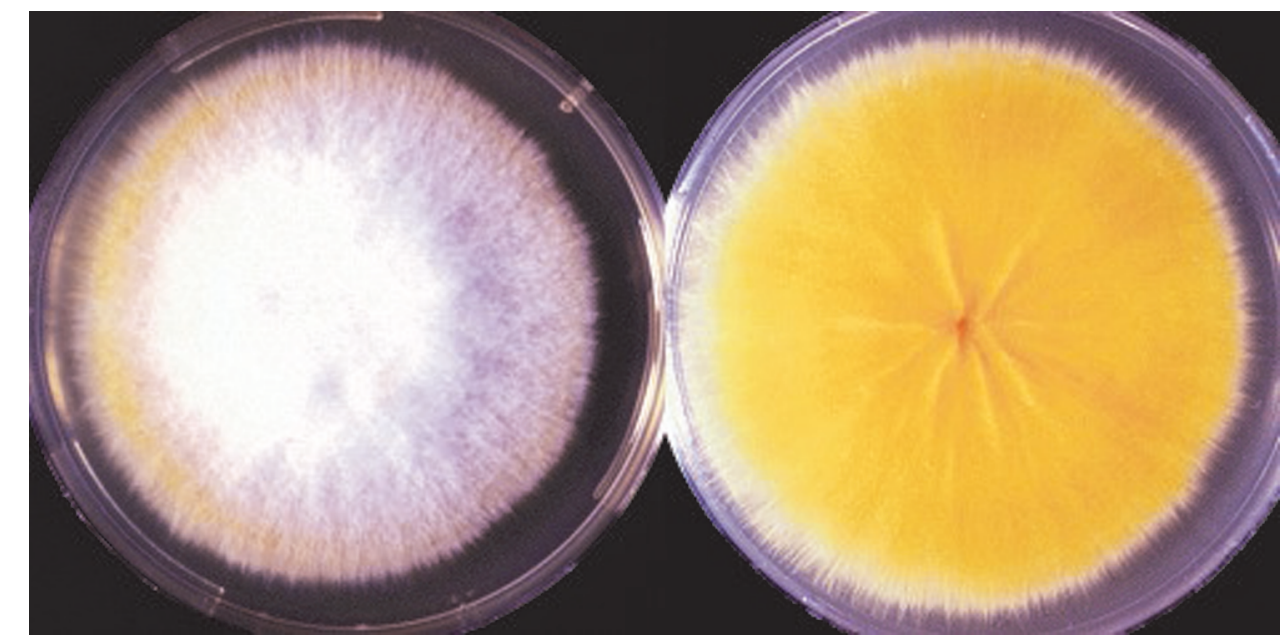

Fig. 5: Culture of Microsporum canis in cultures and cause a diagnostic dilemma. Clinical, radiological, and histopathological correlation is required to reach a definitive diagnosis.

Various culture media and techniques used in the mycology laboratory include Bird seed agar, Cornmeal agar, Czapek Dox agar, Potato dextrose agar, Sabouraud's dextrose agar (SDA) + cycloheximide and antibiotics, and Urea agar with $0.5 \%$ glucose. Automated blood culture systems like BacT Alert can be used to diagnose Candidemia and other fungemia. Sabouraud's dextrose agar is most commonly used in mycology laboratory and is appropriate for the growth of all common yeasts and molds. Bird seed agar is used for Cryptococcus species. Fungi are identified based on colony characteristics on these culture media as well as a microscopic appearance from Gram stain or Lactophenol cotton blue mounts prepared from the colonies. For example, Candida species colonies on SDA are described as white to cream-colored smooth, glabrous, yeast-like. Gram's stain from such colonies reveals gram-positive oval budding yeast cells, whereas colonies of Cryptococcus species are similar to Candida but mucoid in nature. Aspergillus fumigatus colonies on SDA are typically blue-green with a suede-like surface consisting of a dense felt of conidiophores. Lactophenol cotton blue mount from these colonies show uniseriate and columnar conidial heads with the phialides limited to the upper two-thirds of the vesicle and curving to be roughly parallel to each other. ${ }^{9}$

Various tests used for identifying fungi include hair perforation test for Dermatophytes, Chromogenic agar, Germ tube test, 


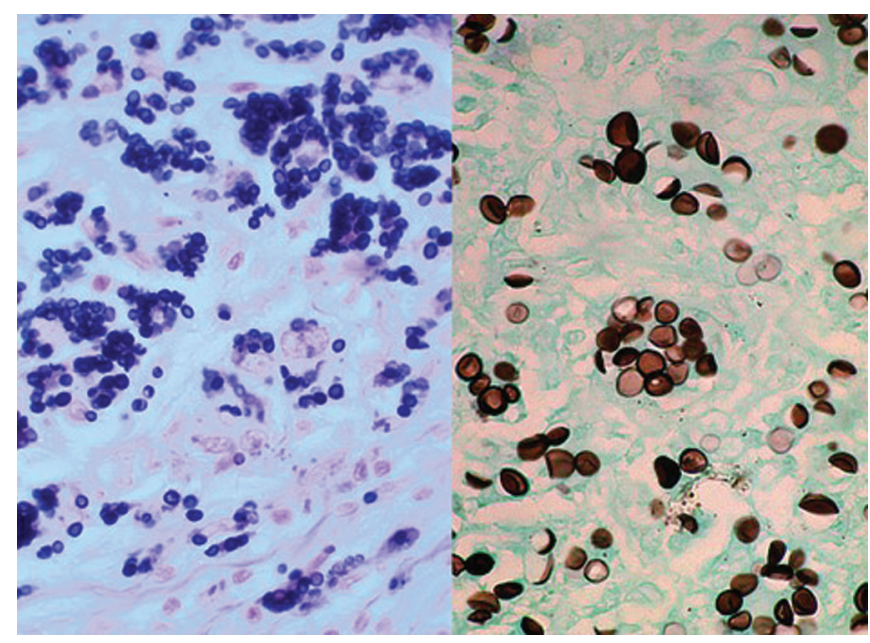

Fig. 6: Tissue morphology of Histoplasma capsulatum showing narrowbased budding yeast cells (Giemsa stain and GMS stain)

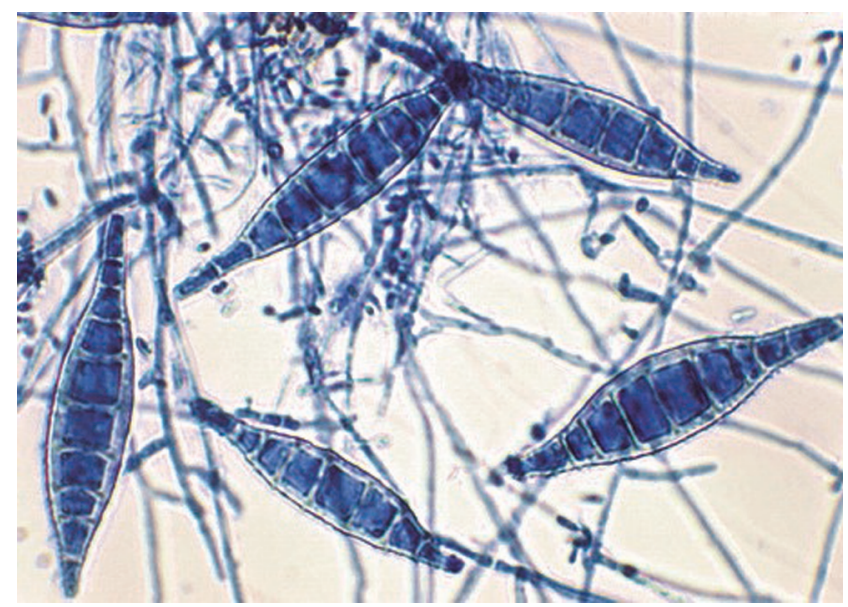

Fig. 8: Spindle-shaped Macroconidia of Microsporum canis (LPCB mount of the colony)

Dalmau plate culture, and carbohydrate assimilation tests used identification of Candida species.

\section{Blood Culture}

Blood culture is a useful investigation for diagnosing fungemia caused by Candida, H. capsulatum, Coccidioides immitis, Blastomyces dermatitidis, and C. neoformans. Trichosporon beigelii is an opportunistic fungal pathogen and Malassezia spp. in children receiving total parenteral nutrition has also been described in various case reports. ${ }^{10,11}$

\section{Automated Identification Systems for FUNGI}

Vitek 2 system by Biomerieux is useful for identifying yeasts like Candida and Cryptococcus. MALDI TOF MS has a wide database for yeasts and molds.

\section{Antifungal Susceptibility Testing}

Broth dilution method of susceptibility testing, E test, and VITEK 2 has been described for antifungal susceptibility testing. These methods are not widely available in microbiology laboratories.

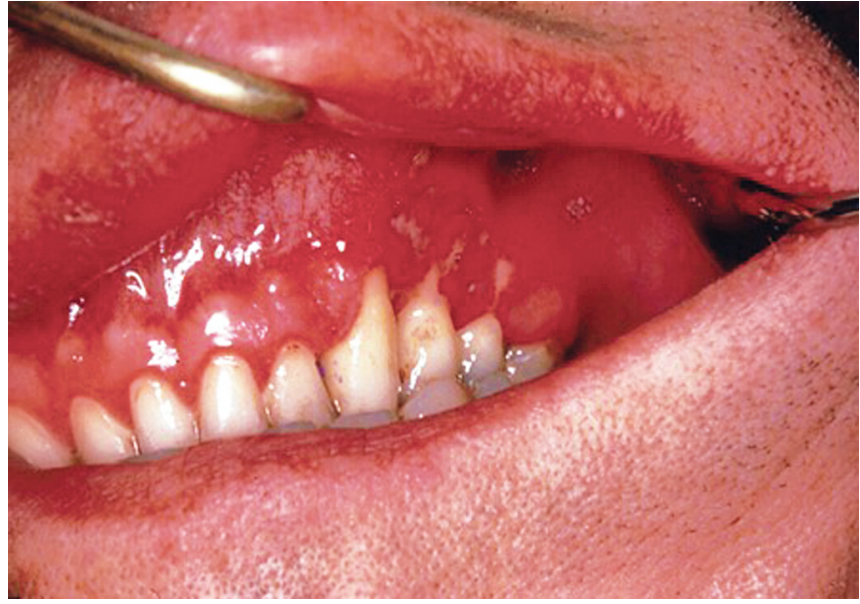

Fig. 7: Histoplasmosis of gum showing ulcer

\section{Role of Serology in the Mycoses DiAgNOSIS}

Enzyme-linked immunosorbent assay (ELISA) for circulating serum galactomannan (GM) and assay for circulating 1-3- $\beta$-D-glucan (BDG) have been used for diagnosis of invasive aspergillosis. The BDG ELISA detects 1,3- $\beta$-D-glucan, which is the major cell wall component of most fungal species, except for fungi of the subdivision Mucormycetes, Cryptococcus spp., and some other Basidiomycota (e.g., Malassezia spp.) that contain less 1,3- $\beta-D-$ glucan in their cell wall and are usually not detected by these tests. Thus, the BDG assay is not specific for the diagnosis of invasive aspergillosis and is also used for the diagnosis of invasive candidiasis. The ELISA for GM detects the circulating GM, a major constituent of Aspergillus cell walls. The sensitivity and specificity of this test may vary considerably according to the patient population and cutoff level used. ${ }^{12,13}$ Serological tests including antigen detection by ELISA and antibody detection by immunodiffusion/ ELISA are described for systemic mycoses like histoplasmosis but it is not widely available in India.

\section{Molecular Diagnostics}

Molecular methods are available for the early diagnosis of invasive fungal infections, multiplex PCR is useful in such cases. European Fungal PCR Initiative group (FPCRI) has standardized and validated protocols for Aspergillus PCR which has a high negative predictive value. SeptiFast PCR is multiplex real-time PCR (Roche Diagnostics, Germany) that detects 20 clinically relevant pathogens including 6 fungi, i.e., 5 Candida spp. and $A$. fumigatus. PCR positivity depends on the site and adequacy of a clinical specimen. ${ }^{2}$

\section{Case Descriptions}

\section{Case 1}

Alaa Al-Juaid et al. described pediatric gastrointestinal basidiobolomycosis in a 5 -year-old child who presented with a prolonged fever of 30-day duration, diarrhea, vomiting, and weight loss. CT scan of the abdomen showed a large retroperitoneal mass with mesenteric lymph node enlargement, suggestive of intestinal lymphoma. He underwent exploratory laparotomy for resection of the mass and a biopsy was taken for 


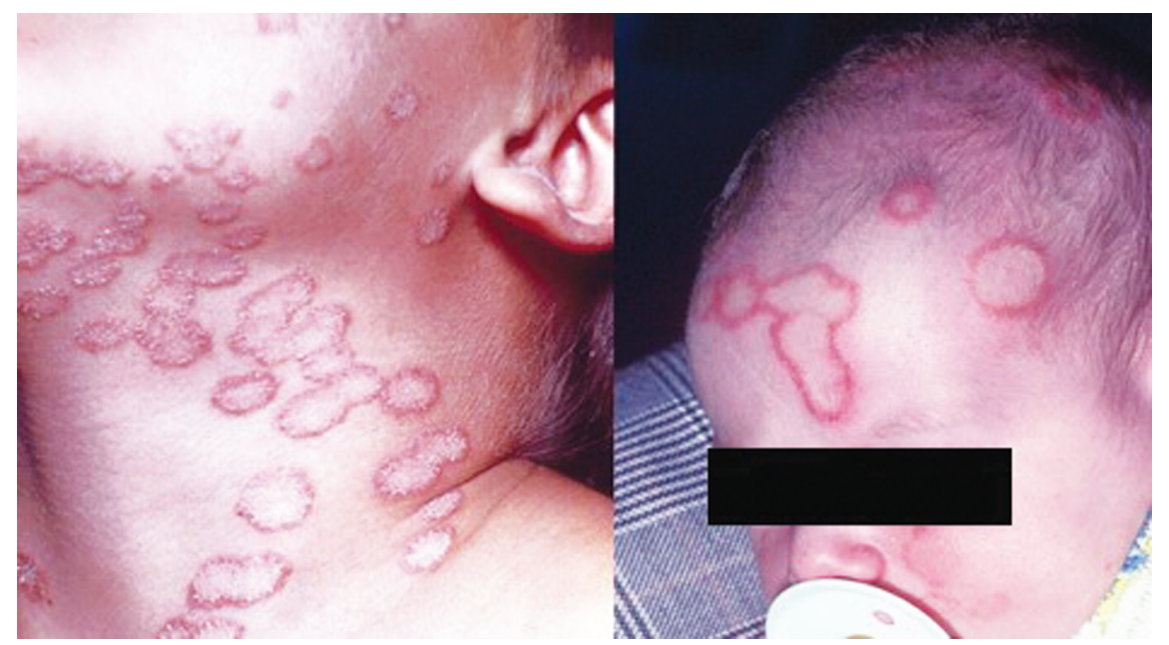

Fig. 9: Dermatophytosis caused by Microsporum species—Clinical presentation

the histopathological examination which identified "SplendoreHoeppli phenomenon" surrounded by oval-shaped organisms with septated hyphae, consistent with colonic basidiobolomycosis. Misdiagnosis as other chronic granulomatous diseases, malignancies, or inflammatory bowel diseases is commonly reported for gastrointestinal basidiobolomycosis. Basidiobolus ranarum is a rare fungal pathogen belonging to Zygomycota (Entomophthorales) and causes unusual chronic skin infections and is increasingly being recognized as a causative agent of gastrointestinal basidiobolomycosis in pediatric populations from tropical and subtropical regions. It causes disease primarily in immunocompetent hosts who present with varied manifestations ranging from abdominal mass and fever with eosinophilia to severe bowel ischemia, necrosis, and shock. ${ }^{14}$

\section{Case 2}

A 11-month-old previously healthy female infant developed fever and emesis followed by convulsion and coma after 2 days. CT displayed subdural hemorrhage in the left tentorium cerebelli and CSF routine tests were normal. During hospitalization, the infant had difficulty breathing and CT showed consolidation in the right lung. Bronchoalveolar lavage fluid (BALF) fungal culture yielded Aspergillus spp. and the GM optical density index in the CSF, in this case, was 3.0, higher than that in the BALF (2.6). Cranial MRI revealed multiple rings reinforced tubercles in sulci. Hence, the patient was clinically diagnosed with CNS aspergillosis and voriconazole was administered. ${ }^{15}$

\section{Case 3}

Maxfield et al. described a case of a 3-year-old child who was diagnosed with acute lymphoblastic leukemia and was initiated on induction chemotherapy. During the course of treatment, the child became febrile with pancytopenia which later progressed to acute kidney injury. The patient was noted to have multiple maculopapular skin lesions involving the trunk and bilateral lower extremities that were suggestive of fungal emboli. Skin biopsies revealed Trichosporon spp. and blood cultures grew T. asahii. Periodic acid Schiff staining from skin biopsy revealed fungal elements including true hyphae, pseudohyphae, arthroconidia, and blastospores. ${ }^{10}$

\section{References}

1. Retrieved from https://mycology.adelaide.edu.au/mycoses/.

2. Seth R, Xess I, Jana M. Diagnosis of invasive fungal infections in children. Indian Pediatr 2019;56(3):229-236. DOI: 10.1007/s13312-0191505-7.

3. Wattier RL, Dvorak CC, Hoffman JA, et al. A prospective, international cohort study of invasive mold infections in children. J Pediatr Infect Dis Soc 2014;4(4):313-322. DOI: 10.1093/jpids/piu074.

4. Kurade SM, Amladi SA, Miskeen AK. Skin scraping and potassium hydroxide mount. Indian J Dermatol Venereol Leprol 2006;72(3):238241. DOI: $10.4103 / 0378-6323.25794$.

5. Abassi M, Boulware DR, Rhein J. Cryptococcal meningitis: diagnosis and management update. Curr Trop Med Rep 2015;2(2):90-99. DOI: 10.1007/s40475-015-0046-y.

6. Azar MM, Hage CA. Laboratory diagnostics for histoplasmosis. J Clin Microbiol 2017;55(6):1612-1620. DOI: 10.1128/JCM.02430-16.

7. Guarner J, Brandt ME. Histopathologic diagnosis of fungal infections in the 21st century. Clin Microbiol Rev 2011;24(2):247-280. DOI: 10.1128/CMR.00053-10.

8. Hageage GJ, Harrington BJ, The use of calcofluor white in clinical mycology. Abstr 269 21st Interscience Conference on Antimicrobial Agents and Chemotherapy, 1981.

9. Retrieved from https://mycology.adelaide.edu.au/descriptions/ hyphomycetes/aspergillus/.

10. Maxfield L, Matthews JJ, Ambrosetti DR, et al. Trichosporon fungemia in a pediatric patient with acute lymphoblastic leukemia. ID Cases 2015;2(4):106-108. DOI: 10.1016/j.idcr.2015.09.007.

11. Al-Sweih N, Ahmad S, Joseph L, et al. Malassezia pachydermatis fungemia in a preterm neonate resistant to fluconazole and flucytosine. Med Mycol Case Rep 2014;5:9-11. DOI: 10.1016/j. mmcr.2014.04.004.

12. Ghosh I, Raina V, Kumar L, et al. Serum galactomannan assay for diagnosis of probable invasive aspergillosis in acute leukemia and hematopoietic stem cell transplantation. Indian J Med Paediatr Oncol 2013;34(2):74-79. DOI: 10.4103/0971-5851.116181.

13. Lamoth F. Galactomannan and 1,3- $\beta$-d-glucan testing for the diagnosis of invasive aspergillosis. J Fungi (Basel). 2016;2(3):22. DOI: 10.3390/jof2030022.

14. Al-Juaid A, Al-Rezqi A, Almansouri W, et al. Pediatric gastrointestinal basidiobolomycosis: case report and review of literature. Saudi J Med Med Sci 2017;5(2):167-171.

15. Wang Y, Li D, Qiao L, et al. Infant central nervous system aspergillosis with first-episode of intracranial hemorrhage: a case report. Medicine (Baltimore) 2017;96(47):e8893. DOI: 10.1097/MD.0000000000008893. 